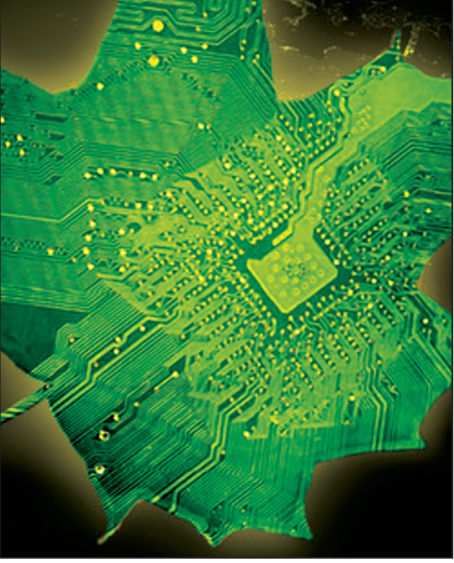

\title{
Splitting $\mathrm{CO}_{2}$ to produce syngas and hydrocarbon fuels: PEC and STC
}

\author{
By Eva Karatairi \\ Feature Editor James E. Miller
}

T hough we live in the Anthropocene era, growing awareness of the potentially damaging global consequences of human dominance is prompting efforts to achieve and retain a favorable global concentration and dynamic equilibrium for $\mathrm{CO}_{2}$, wherein production and consumption balance one another. Large-scale production of sustainable hydrocarbon fuels is a crucial piece of this balancing act, and to this end, scientists and engineers are manipulating three widely available ingredients - sunlight, water, and $\mathrm{CO}_{2}$ - through electrochemical, photoelectrochemical, and solar thermochemical means. The focus of much of this work is the initial production of syngas, a versatile precursor of synthetic fuels, consisting of $\mathrm{CO}$ and $\mathrm{H}_{2}$, which leverages established commercial processes for syngas conversion to hydrocarbon fuels. In the previous issue of EQ, global efforts on the most technologically ready approach of the three, electrolysis, were examined. Herein, we take a closer look at photoelectrochemistry (PEC) and solar thermochemistry (STC) in this context.

PEC, like electrolysis, exploits energetic electrons to provide the energy and to drive the chemical conversions. The difference is the more direct coupling and integration of the electron source, electrode/catalysts, and other components in PEC. For example, since October 2015, the Joint Center for Artificial Photosynthesis (JCAP, a US DOE Energy Innovation Hub) has conducted the mesoscale design project, which is focused on creating a fully integrated PEC device with improved efficiency for the production of renewable fuels. The device comprises a combined PV and electrolyzer, with a catalyst deposited directly on top of solar photon absorbers. It requires the assembly of photoactive semiconductors, cathodic and anodic catalysts, and an ion-conducting membrane.

David Tiede, member of the Argonne-Northwestern Solar Energy Research Center, explained the approach: "In classical electrolysis, the electrode is agnostic; it doesn't know where it gets potential from.... PEC involves semiconductors which, when you shine light on them, move electrons up from the valence to the conduction band. These become mobile electrons, with a reducing power generated by light." The energetic mobile electrons (and/or holes) can move to the surface or interface regions of the device where they are available for reaction. Tiede added that the initial light absorption pushes electrons to highly exited orbitals, yielding the so-called hot carriers. "If captured in less than picoseconds, before they lose their energy in the form of heat and end up in the lowest excited states, then one has very reactive hot electrons," he said.

The efficiencies reported for PEC vary, but many are impressive. Earlier this year, the team of Michael Graetzel at École Polytechnique Fédérale de Lausanne (EPFL) in Switzerland reported a targeted electrochemical transformation of $\mathrm{CO}_{2}$ to $\mathrm{CO}$ and oxygen, using $\mathrm{CuO}$ nanowires covered with $\mathrm{SnO}_{2}$, by means of atomic layer deposition. With the additional use of a $\mathrm{GaInP} / \mathrm{GaInAs} / \mathrm{Ge}$ photovoltaic, they achieved the solar-driven splitting of $\mathrm{CO}_{2}$ at an efficiency of $13.4 \%$.

Still many challenges, particularly around materials, remain, as lifetimes of PEC systems tend to be short (measured in hours or days), and the demonstrations are at laboratory scale. "PEC approaches must focus on longevity of the components and design of complete, practical, and scalable demonstration systems," said Sophia Haussener, professor at EPFL, who specializes in both PEC and STC.

Design of catalysts for improved efficiency and selectivity is an area of active interest. Zetian Mi, from McGill University in Montreal, explained why syngas is an attractive target for PEC. "Compared to the one-step direct reduction of $\mathrm{CO}_{2}$ and $\mathrm{H}_{2} \mathrm{O}$ to liquid hydrocarbons and methanol by a multi-electron transfer process, it is more feasible to take a two-step process: First reduce $\mathrm{CO}_{2}$ and $\mathrm{H}_{2} \mathrm{O}$ by a two-electron pathway to syngas intermediate, which will then be converted to liquid hydrocarbons and methanol using well-established standard industrial processes, like Fischer-Tropsch (FT) technology," he said.

Mi, together with Sheng Chu, also from McGill University, and others recently reported syngas production in an aqueous photoelectrochemical cell, with the desirable $\mathrm{CO} / \mathrm{H}_{2}$ ratio of $1: 2$. "We found that $\mathrm{Cu}$ and $\mathrm{ZnO}$, a classical combination in industrial $\mathrm{CO}_{2}$ hydrogenation, could work cooperatively and efficiently to achieve a tunable syngas composition that meets the requirements for different downstream products," said Mi. He added that boosting the reduction selectivity into $\mathrm{CO}$ was one of the biggest challenges they had to address, because of the domination of the $\mathrm{H}_{2}$ evolution reaction in an aqueous solution.

"Right now, the best catalyst we know is made of $\mathrm{Cu}$," said Jens Nørskov, professor of chemical engineering and photon science at Stanford University and director of SUNCAT. "In our lab, we are working both on understanding $\mathrm{Cu}$ [catalysts], as well as on new designs that will work better," he said. Nørskov wants to 
skip the extra step and is looking for a way to electrochemically transform $\mathrm{CO}_{2}$ to something more reduced. "We don't have the catalysts that enable this efficiently. We do have catalysts, based on $\mathrm{Cu}$ in particular, that work, and we can make a number of interesting products. What we want is to make these catalysts work with less energy input ... and also to be able to control their selectivity. Make only one or a few products rather than a whole soup of them," he added.

Unlike electrochemistry (EC) and PEC, in STC processes, syngas is thermally produced using concentrating solar power systems - special mirror assemblies that track the sun and concentrate the rays to high intensities. In a typical two-step cyclic STC process, solar-thermal heat reduces a metal oxide in a first step, releasing oxygen, and $\mathrm{H}_{2}$ and/or $\mathrm{CO}$ are produced in a second distinct step when the oxide is reoxidized by $\mathrm{H}_{2} \mathrm{O}$ and/or $\mathrm{CO}_{2}$, respectively.

For both steps to be thermodynamically and kinetically feasible, metal oxides that reduce at very high temperatures tend to be preferred. Oxides containing iron $(\mathrm{Fe})$, zinc $(\mathrm{Zn})$, zirconium $(\mathrm{Zr})$, and cerium $(\mathrm{Ce})$ are the spearheads in this area. While materials development and reactor engineering efforts have decreased reduction temperatures to less than $1500^{\circ} \mathrm{C}$, operating temperatures well above $1200^{\circ} \mathrm{C}$ are still common. Nonetheless, compared to PEC, "STC approaches are more successful in stability (in the range of months) and have been demonstrated at scales of kilowatts to hundreds of kilowatts," said Haussener.

The European Commission-supported SOLAR-JET project, led by Aldo Steinfeld and Philipp Furler of ETH Zürich, highlighted the entire production chain for renewable kerosene by solar thermochemical splitting of $\mathrm{CO}_{2}$ and $\mathrm{H}_{2} \mathrm{O}$. In this case, nonstoichiometric ceria $\left(\mathrm{CeO}_{2-\delta}\right)$, in the form of a reticulated porous ceramic structure, was used as the reactive oxide in a high-temperature two-step $\mathrm{H}_{2} \mathrm{O} / \mathrm{CO}_{2}$ thermochemical splitting reactor. The researchers reported conducting 291 consecutive $\mathrm{CO}_{2}$ and $\mathrm{CO}_{2} / \mathrm{H}_{2} \mathrm{O}$ splitting cycles, yielding 700 standard liters of syngas. The collected syngas was subsequently processed via FT and hydrocracking at Shell Global Solutions in Amsterdam, giving liquid hydrocarbons with a composition of $17.1 \mathrm{wt} \%$ naphtha, $35.6 \mathrm{wt} \%$ kerosene, $17.1 \mathrm{wt} \%$ gasoil, and $30.2 \mathrm{wt} \%$ of heavier fractions.

Though very high efficiencies are within reach theoretically, demonstrated efficiencies are currently in the range of 5\%. Efficiencies are currently limited by both engineering (reactor design) and materials aspects, and the interplay between the two. The materials aspects of the challenge are becoming ever more appreciated. In Haussener's view, "STC needs to be more focused on materials with better redox behavior" (deeper reduction at lower temperatures and higher oxygen partial pressure, while maintaining oxidation reactivity and rapid kinetics).

Materials work of this type is being pursued around the world, much of it now focused on $\mathrm{Ce}$ and redox active perovskites. One of the first successful uses of nonstoichiometric perovskite oxides for STC $\mathrm{CO}_{2}$ (and water) splitting was demonstrated at Sandia National Laboratories. $\mathrm{LaAlO}_{3}$ doped with $\mathrm{Mn}$ and $\mathrm{Sr}$ efficiently split $\mathrm{CO}_{2}$, producing significantly more fuel than $\mathrm{Ce}$ at reduction temperatures well below $1550^{\circ} \mathrm{C}$ and maintaining constant activity for many cycles. Computational design and discovery techniques now focus on similar materials for STC.

Additionally, many groups are pursuing a variety of hybrid approaches that incorporate aspects of STC with chemical looping that can help advance the field. For example, Fanxing Li at North Carolina State University and his team recently reported work on perovskite-structured oxides $\mathrm{Sr}_{2} \mathrm{Fe}_{2} \mathrm{O}_{5}$ and $\mathrm{Sr}_{3} \mathrm{Fe}_{2} \mathrm{O}_{6}$ dispersed in an inert and earth-abundant $\mathrm{Ca}_{0.5} \mathrm{Mn}_{0.5} \mathrm{O}$ phase to yield nanocomposites with excellent stability. The materials were first reduced by methane at 900 $980^{\circ} \mathrm{C}$ to yield syngas and then reoxidized with $\mathrm{CO}_{2}$ (with almost complete $\mathrm{CO}_{2}$ conversion) to provide additional $\mathrm{CO}$; the overall process was proposed as a route to acetic acid with greatly decreased $\mathrm{CO}_{2}$ emissions.

Predictions are not easy for the when and how of sustainable fuels or, more broadly, chemicals. Nørskov noted, "It is really at the heart of the future in many ways, because we need fuels but we also need chemicals. A lot of the materials today are based on the carbon polymer industry, which is based on fossil input. If we want to change that in the future, we need processes with which we can make these molecules in a sustainable fashion."

For now, EC, PEC, and STC research complement one another and mitigate risk, each having different challenges to address and different solutions to offer. Electrolysis has already left the lab and seems closer to succeeding in the goal of providing the transportation fleet with sustainable synthetic fuels with no fossil origins. STC has not demonstrated the efficiencies of PEC, but PEC has not demonstrated the robustness of STC. In any case, as Haussener and Mi said, success will also depend on government support and the early involvement of industry. "Policy actions like a carbon tax will ensure a plane playfield for the competition of renewable fuel and fossil fuel approaches," said Mi. When asked to make a prediction, Tiede said, "We are trying to compete against natural fuels and gas, and it is of course cheaper to take these materials from the ground than to create it from scratch. It depends how much people like and need clean and sustainable versus soon-to be depleted geological sources. It might take long to develop a technology at scale, but we will need it eventually. It will mature, get better, and pretty soon will grow. So, I suspect, this is how it begins." 